\title{
On the Transfer of Information and Energy in Multi-User Systems
}

\author{
Ali Mohammad Fouladgar, Student Member, IEEE, and Osvaldo Simeone, Member, IEEE
}

\begin{abstract}
The problem of joint transfer of information and energy for wireless links has been recently investigated in light of emerging applications such as RFID and body area networks. Specifically, recent work has shown that the additional requirements of providing sufficient energy to the receiver significantly affects the design of the optimal communication strategy. In contrast to most previous works, this letter focuses on baseline multi-user systems, namely multiple access and multihop channels, and demonstrates that energy transfer constraints call for additional coordination among distributed nodes of a wireless network. The analysis is carried out using informationtheoretic tools, and specific examples are worked out to illustrate the main conclusions.
\end{abstract}

Index Terms-Energy transfer, information theory, multiple access channel, multi-hop channel, energy-harvesting.

\section{INTRODUCTION}

Electromagnetic waves carry both energy and information. Information is modulated on the amplitude and phase of an electromagnetic wave, while energy transfer is realized via either near-field induction or far-field radiation. Applications of wireless energy transfer include passive radio-frequency identification (RFID) [1], body area networks [2], and power beaming using microwaves or laser from satellites or aircraft [3].

Recent research has recognized that the two tasks of energy and information pose conflicting constraints on the design of a wireless system [4]-[7]. This can be easily understood by noting that the power of a signal depends on its average squared value, while the quantity of information depends on the amount of "variations", or more specifically on the entropy rate, of the signal itself - maximizing one generally does not lead to a maximum of the other.

Previous work [4]-[7] has focused on point-to-point or broadcast systems, and specifically on the problem of maximizing the information rate subject to minimum received energy constraints. Reference [4] studied a single point-topoint channel, [5], [7] investigated a set of parallel point-topoint channels and [6] considered a multi-antenna broadcast channel. It was shown that, as the argument above suggests, the design of the optimal transmission strategy depends drastically on the received requirements. Incidentally, we note that the setting at hand leads to very different insights with respect to the problem of maximizing the information rate subject to

A. M. Fouladgar and O. Simeone are with the Center for Wireless Communications and Signal Processing Research (CWCSPR), ECE Department, New Jersey Institute of Technology (NJIT), Newark, NJ 07102, USA (email: af82@njit.edu, osvaldo.simeone@njit.edu).

The work of O. Simeone was supported in part by the U.S. National Science Foundation under Grant No. 0914899.

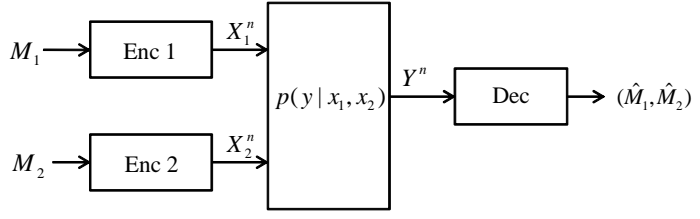

Figure 1. DM-MAC with independent messages and received energy constraints.

maximum received energy constraints considered in 8 1]. We also remark that the model considered in [9] is different in that it assumes that energy can be transferred between two nodes via a separate channel devoted to energy transfer.

While previous work focused on systems with a single transmitter, in this letter we take a first look at systems with multiple transmitters by focusing on the two baseline scenarios of multiple access channels (Fig. 11) and multi-hop channels (Fig. 2). The main aim of this study is to argue that novel forms of coordination among distributed transmitters of a wireless networks become useful when the system design has to account for energy transfer requirements. More specifically, our contributions are as follows.

- Multiple access channel with received energy constraint: In Sec. III we consider the standard multiple access channel in Fig. 11 with the additional constraint that the energy received by the decoder be large enough. First, the characterization of all the achievable trade-off among rate pairs and received energy is obtained, extending the point-to-point result of [4]. Then, an example is provided that demonstrates the enhanced need for coordination between the two encoders in order to satisfy the requirement on energy transfer;

- Multi-hop channel with a harvesting relay: In Sec. III, we turn our attention to the multi-hop channel in Fig. 2. where the relay is assumed to be able to harvest the energy received from the encoder for transmission to the decoder. A characterization of the capacity is derived. An example is then described that illustrates the novel issues that arise in the design of the communication strategy in the first hop due to the harvesting capabilities for transmission over the second hop.

\footnotetext{
${ }^{1}$ As an instance of this fact, one can compare [5] Theorem 1] with [8 Theorem 2].
} 


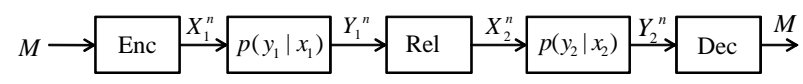

Figure 2. DM-MHC with a relay that can harvest the received energy.

\section{Multiple Access Channel With Received ENERGY CONSTRAINT}

In this section, we consider a Discrete Memoryless Multiple Access Channel (DM-MAC) $\left(\mathcal{X}_{1} \times \mathcal{X}_{2}, p\left(y \mid x_{1}, x_{2}\right), \mathcal{Y}\right)$ in which two encoders wish to communicate independent messages to the decoder and at the same time, to provide the latter with sufficient received energy (see Fig. 11). The channel is described by three finite alphabets $\mathcal{X}_{1}, \mathcal{X}_{2}, \mathcal{Y}$, which are subsets of real numbers, and a collection of conditional probability mass functions (pmfs) $p\left(y \mid x_{1}, x_{2}\right)$ on $\mathcal{Y}$. All definitions are standard, see, e.g., [10, Chapter 4], except for the requirement on the received energy to be discussed below. We use the same notation as [10].

Specifically, a $\left(2^{n R_{1}}, 2^{n R_{2}}, P_{1}, P_{2}, n\right)$ code for the DMMAC consists of:

- two message sets $2\left[1: 2^{n R_{1}}\right]$ and $\left[1: 2^{n R_{2}}\right]$;

- two encoders, where encoder 1 assigns a codeword 3 $x_{1}^{n}\left(m_{1}\right)$ to each message $m_{1} \in\left[1: 2^{n R_{1}}\right]$ and encoder 2 assigns a codeword $x_{2}^{n}\left(m_{2}\right)$ to each message $m_{2} \in\left[1: 2^{n R_{2}}\right]$. We have the input cost constraints

$$
c_{k}^{n}\left(x_{k}^{n}\left(m_{k}\right)\right)=\frac{1}{n} \sum_{i=1}^{n} c_{k}\left(x_{k i}\left(m_{k}\right)\right) \leq P_{k}
$$

for given functions $c_{k}: \mathcal{X} \rightarrow \mathbb{R}_{+}$, for all messages $m_{k} \in$ $\left[1: 2^{n R_{k}}\right]$, and $k=1,2$;

- a decoder that assigns an estimate $\left(\hat{m}_{1}, \hat{m}_{2}\right) \in[1$ : $\left.2^{n R_{1}}\right] \times\left[1: 2^{n R_{2}}\right]$ to each received sequence $y^{n}$.

The message pair $\left(M_{1}, M_{2}\right)$ is uniformly distributed in the set $\left[1: 2^{n R_{1}}\right] \times\left[1: 2^{n R_{2}}\right]$. The average probability of error is defined as $P_{e}^{(n)}=\operatorname{Pr}\left\{\left(\hat{M}_{1}, \hat{M}_{2}\right) \neq\left(M_{1}, M_{2}\right)\right\}$. The received energy for a sequence $y^{n}$ is defined as

$$
b^{n}\left(y^{n}\right)=\frac{1}{n} \sum_{i=1}^{n} b\left(y_{i}\right)
$$

for a given function $b: \mathcal{Y} \rightarrow \mathbb{R}_{+}$. A rate-energy triple $\left(R_{1}, R_{2}, B\right)$ is said to be achievable with energy cost constraints $\left(P_{1}, P_{2}\right)$ for the DM-MAC if there exists a sequence of $\left(2^{n R_{1}}, 2^{n R_{1}}, P_{1}, P_{2}, n\right)$ codes such that

$$
\limsup _{n \rightarrow \infty} P_{e}^{(n)}=0
$$

and

$$
\limsup _{n \rightarrow \infty} \operatorname{Pr}\left[\frac{1}{n} \sum_{i=1}^{n} b\left(Y_{i}\right)<B-\epsilon\right]=0 .
$$

for any $\epsilon>0$. Condition (4) states that the average received energy should be at least $B$ with high probability as $n$ grows $\operatorname{larg} 4$. The capacity-energy region $\mathcal{C}_{e}\left(P_{1}, P_{2}\right)$ of the DMMAC is the closure of the set of achievable rate-energy triple $\left(R_{1}, R_{2}, B\right)$.

\footnotetext{
${ }^{2}[1: n]=\{1, \ldots, n\}$ for any integer $n$.

${ }^{3}$ We denote $X^{n}$ as the sequence $X^{n}=\left[X_{1}, \ldots, X_{n}\right]$.

${ }^{4}$ This entails also the weaker constraint $1 / n \sum_{i=1}^{n} \mathrm{E}\left[b\left(Y_{i}\right)\right] \geq B-\epsilon$, for $n$ large enough (see Appendix A).
}

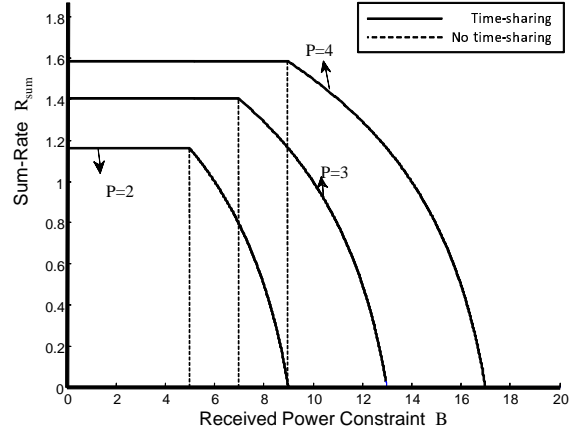

Figure 3. Sum-rate $R_{\text {sum }}$ versus received energy constraint $B$ for the Gaussian MAC studied in Sec. [II-B

\section{A. Capacity-Energy Region}

We are now ready to present a characterization of the DMMAC capacity-energy region.

Theorem 1. The capacity-energy region $\mathcal{C}_{e}\left(P_{1}, P_{2}\right)$ of the $D M-M A C$ with received energy constraint is the union of the set of all rate-energy triples $\left(R_{1}, R_{2}, B\right)$ such that the inequalities

$$
\begin{aligned}
R_{1} & \leq I\left(X_{1} ; Y \mid X_{2}, Q\right), \\
R_{2} & \leq I\left(X_{2} ; Y \mid X_{1}, Q\right), \\
R_{1}+R_{2} & \leq I\left(X_{1}, X_{2} ; Y \mid Q\right), \\
\text { and } B & \leq E[b(Y)]
\end{aligned}
$$

hold for some pmfs $p(q), p\left(x_{1} \mid q\right)$ and $p\left(x_{2} \mid q\right)$ satisfying the constraints $E\left[c\left(X_{k}\right)\right] \leq P_{k}$, for $k=1,2$. The alphabet of $Q$ can be bounded as $|\mathcal{Q}| \leq 4$.

It is recalled, for future reference, that the variable $Q$ in (5) enables time-sharing between different pairs of codebooks used by the two encoders. Specifically, when $Q=q$ for some $q \in \mathcal{Q}$, the codebooks to be used by the encoders are characterized by the conditional pmfs $p\left(x_{1} \mid q\right)$ and $p\left(x_{2} \mid q\right)$ as per standard random coding arguments. We remark that time-sharing requires coordination between the encoders that have to agree on a sequence $Q^{n}$ and switch to the preassigned codebooks when appropriate.

Proof: Achievability and converse follow with minor variations from standard results on the DM-MAC (see [10, Chapter 4]). Specifically, achievability follows from the same random coding arguments in [10, Chapter 4]. The only difference is that, in order to guarantee the constraint (4), an additional error event is added to the conventional analysis in [10, Chapter 4] for the case in which the event within square brackets in (4) does not hold. This event is immediately shown to have vanishing probability for large $n$ by the weak law of large numbers. Some details on the converse can be found in Appendix A.

\section{B. Example}

In order to illustrate the novel aspects in the system design that are entailed by the minimum receive energy constraint (4), 
we now consider the Gaussian MAC $Y=X_{1}+X_{2}+Z$, where $Z \sim \mathcal{N}(0,1)$ is the additive noise. Let the input cost function be $c_{k}\left(x_{k}\right)=x_{k}^{2}$ for $k=1,2$ and the received energy function be $b(y)=y^{2}$. Note that the Gaussian MAC is not DM, but the result in Theorem 1 applies as per standard arguments [10. Sec. 3.4]. From Theorem 1, all achievable sum-rates for the Gaussian MAC at hand can be written as

$$
R_{\text {sum }}=I\left(X_{1}, X_{2} ; Y \mid Q\right),
$$

for some pmfs $p(q), p\left(x_{1} \mid q\right)$ and $p\left(x_{2} \mid q\right)$ under the constraints $\mathrm{E}\left[Y^{2}\right] \geq B$ and $\mathrm{E}\left[X_{k}^{2}\right] \leq P_{k}$, for $k=1,2$.

As it is well known (see, e.g., [10]), without any constraint on the received energy, the maximum sum-rate is given as

$$
R_{\text {sum }}=\frac{1}{2} \log _{2}(1+2 P),
$$

which is achieved by setting $Q$ to a constant and $X_{k} \sim$ $\mathcal{N}(0, P)$ for $k=1,2$. In other words, maximum information transfer does not require time sharing. Moreover, with this choice, the received power is $E\left[Y^{2}\right]=2 P+1$. Therefore, if $B \leq 2 P+1$, then, even under the received energy constraint (4), the maximum sum-rate is given by (7) and time sharing is not needed.

Now we assume that $B>2 P+1$, and consider a time-sharing strategy whereby $Q \sim \operatorname{Ber}(\lambda)$ and $X_{1}, X_{2} \sim$ $\mathcal{N}\left(0, P^{\prime}\right)$ for $Q=1$ and $X_{1}=X_{2}=\sqrt{P^{\prime \prime}}$ for $Q=0$ for some $0 \leq \lambda \leq 1, P^{\prime} \geq 0$ and $P^{\prime \prime} \geq 0$. In other words, for $Q=1$ information-maximizing codebooks are used, while for $Q=0$ the two encoders transmit the constant signals $X_{1}=X_{2}=\sqrt{P^{\prime \prime}}$. The latter choice maximizes the energy transfer due to coherent combining at the receiver, but carries no information. Optimizing over the parameters $\left(P^{\prime}, P^{\prime \prime}, \lambda\right)$, from (6), the following sum-rate is achievable

$$
\begin{aligned}
R_{\text {sum }}=\underset{0 \leq \lambda \leq 1, P^{\prime \prime}, P^{\prime \prime}}{\operatorname{maximize}} \quad \frac{\lambda}{2} \log _{2}\left(1+2 P^{\prime}\right) \\
\text { subject to } \lambda P^{\prime}+(1-\lambda) P^{\prime \prime} \leq P, \\
B \leq 2 \lambda P^{\prime}+4(1-\lambda) P^{\prime \prime}+1 .
\end{aligned}
$$

This rate is shown in Figure 3 versus the constraint on the received energy $B$ for different values of $P$ along with the sum-rate (7) obtained with no time-sharing. It is seen that, as the received energy constraint $B$ becomes large enough (i.e., $B>2 P+1)$, time-sharing is necessary to achieve the optimal performance. This demonstrates that additional coordination is generally needed between the encoders in order to obtain the desired trade-off between information and energy transfer.

\section{Multi-Hop Channel With a Harvesting Relay}

In this section, we consider the three-node Discrete Memoryless Multi-Hop Channel (DM-MHC) in Figure 2, in which the encoder wishes to communicate a message $M$ to the decoder with the help of a relay. The relay can harvest the energy received from the encoder as formalized below. We refer to the relay as having energy-harvesting capabilities. The DM-MHC is characterized by two separate DM pointto-point channels $\left(\mathcal{X}_{1}, p\left(y_{1} \mid x_{1}\right), \mathcal{Y}_{1}\right)$ and $\left(\mathcal{X}_{2}, p\left(y_{2} \mid x_{2}\right), \mathcal{Y}_{2}\right)$. All definitions are standard, see, e.g., [10, Chapter 16], except for the fact that the relay can harvest energy from the received signal.
Specifically, a $\left(2^{n R}, P_{1}, P_{2}, n\right)$ code for the DM-MHC consists of

- a message set $\left[1: 2^{n R}\right]$;

- an encoder that assigns a codeword $x_{1}^{n}(m)$ to each message $m \in\left[1: 2^{n R}\right]$. We have the input cost constraint

$$
c_{1}^{n}\left(x_{1}^{n}(m)\right)=\frac{1}{n} \sum_{i=1}^{n} c_{1}\left(x_{1 i}(m)\right) \leq P_{1}
$$

for some function $c_{1}: \mathcal{X}_{1} \rightarrow \mathbb{R}_{+}$, and for all messages $m \in\left[1: 2^{n R}\right]$;

- a relay encoder that assigns a symbol $x_{2 i}\left(y_{1}^{i-1}\right)$ to each past received sequence $y_{1}^{i-1} \in \mathcal{Y}_{1}^{i-1}$ for each time $i \in$ $[1: n]$. The relay input is constrained so that condition

$$
\frac{1}{n} \sum_{i=1}^{n} c_{2}\left(x_{2 i}\right) \leq \frac{1}{n} \sum_{i=1}^{n} b\left(y_{1 i}\right)+P_{2}
$$

is satisfied. This implies that the input cost $\frac{1}{n} \sum_{i=1}^{n} c_{2}\left(x_{2 i}\right)$ should be less than the average harvested energy $\frac{1}{n} \sum_{i=1}^{n} b\left(y_{1 i}\right)$ and the available power $P_{2}$;

- a decoder that assigns an estimate $\hat{m} \in\left[1: 2^{n R}\right]$ to each received sequence $y_{2}^{n}$.

We assume that the message $M$ is uniformly distributed in the set $\left[1: 2^{n R}\right]$. The average probability of error is defined as $P_{e}^{(n)}=\operatorname{Pr}\{\hat{M} \neq M\}$. A rate $R$ is said to be achievable with energy cost constraints $P_{1}, P_{2}$ if there exists a sequence of $\left(2^{n R}, P_{1}, P_{2}, n\right)$ codes such that (3) is satisfied. The capacityenergy function $\mathcal{C}_{e}\left(P_{1}, P_{2}\right)$ of the DM-MHC is the supremum of the set of all achievable rates $R$.

\section{A. Capacity-Energy Function}

We now present a characterization of the DM-MHC capacity-energy function.

Theorem 2. The capacity-energy function $\mathcal{C}_{e}\left(P_{1}, P_{2}\right)$ of the DM-MHC with a harvesting relay is

$$
\begin{array}{r}
\mathcal{C}_{e}\left(P_{1}, P_{2}\right)=\max _{p\left(x_{1}\right): E\left[c_{1}\left(X_{1}\right)\right] \leq P_{1}} \min \left\{I\left(X_{1} ; Y_{1}\right),\right. \\
p\left(x_{2}\right): E\left[\max _{2}\left(X_{2}\right)\right] \leq E\left[b\left(Y_{1}\right)\right]+P_{2} \\
\left.I\left(X_{2} ; Y_{2}\right)\right\} .
\end{array}
$$

Proof: The achievability follows via decode-and-forward using the same arguments as in [10, Ch. 16]. The only difference is that, in order to guarantee that condition (10) is satisfied, an error event is introduced for the case where (10) does not hold, that is shown to have vanishing probability as $n \rightarrow \infty$ by the weak law of large numbers. Some details on the converse can be found in Appendix B.

\section{B. Example}

Consider a noiseless channel $Y_{1}=X_{1}$ with $\mathcal{X}_{1}=\mathcal{Y}_{1}=$ $\{-2,-1,1,2\}$ followed by a Gaussian channel $Y_{2}=X_{2}+Z$, with $Z \sim \mathcal{N}\left(0, N_{0}\right)$. Note that the second channel is not DM, but the analysis applies using conventional arguments [10, Sec. 3.4]. Let the input cost functions be $c_{k}\left(x_{k}\right)=x_{k}^{2}$ for $k=1,2$ and the received energy function be $b\left(y_{1}\right)=y_{1}^{2}$. 

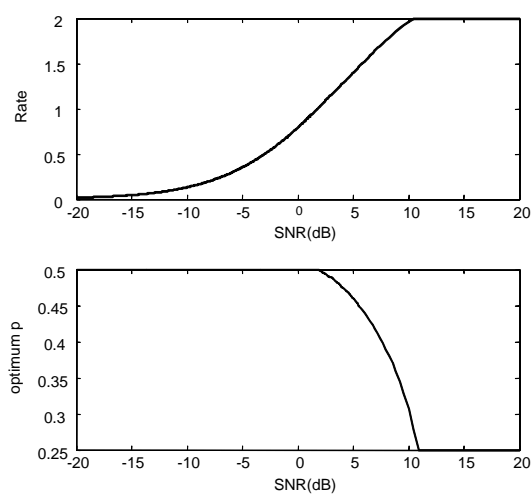

Figure 4. Capacity-energy $\mathcal{C}_{e}\left(P_{1}, P_{2}\right)$ versus the SNR (upper figure) and optimum probability $p$ (lower figure) for the multi-hop channel studied in Sec. III-B $\left(P_{1}=4, P_{2}=0\right)$.

We first observe that, if $b\left(y_{1}\right)=0$ for all $y_{1} \in$ $\{-2,-1,1,2\}$ and thus no energy can be harvested at the relay, then the capacity is given by $\min \left\{2,1 / 2 \log _{2}\left(1+P_{2}\right)\right\}$, which is achieved by setting $X_{1}$ to be uniformly distributed in the set $\{-2,-1,1,2\}$ and $X_{2} \sim \mathcal{N}\left(0, P_{2}\right)$. Note that, in this conventional case, the codebook selected by the encoder depends on the quality of the second link only through the rate, which needs to be set to $C$, but is otherwise independent since $X_{1}$ is uniformly distributed irrespective of the quality of the second hop.

We now consider the effect of a harvesting relay. From Theorem 2, and using the symmetry of the problem, the capacity-energy function can be written as

$$
\begin{aligned}
\mathcal{C}_{e}\left(P_{1}, P_{2}\right) & =\max _{\substack{0 \leq p \leq 1 / 2: \\
6 p+1 \leq P_{1}}}\left\{-2 p \log _{2} p-(1-2 p) \log _{2}\left(\frac{1}{2}-p\right)\right. \\
& \left.\frac{1}{2} \log _{2}\left(1+\frac{P_{2}+6 p+1}{N_{0}}\right)\right\} .
\end{aligned}
$$

This is obtained by setting $p=\operatorname{Pr}\left[X_{1}=2\right]=\operatorname{Pr}\left[X_{1}=\right.$ $-2]$ and $1 / 2-p=\operatorname{Pr}\left[X_{1}=1\right]=\operatorname{Pr}\left[X_{1}=-1\right]$ and $X_{2} \sim \mathcal{N}\left(0, P_{2}+\mathrm{E}\left[Y_{1}^{2}\right]\right)$ given that $\mathrm{E}\left[Y_{1}^{2}\right]=\mathrm{E}\left[X_{1}^{2}\right]=$ $2\left(4 p+\left(\frac{1}{2}-p\right)\right)=6 p+1$. The capacity and the optimum value of $p$ are shown in Figure 4 versus the signal-to-noise ratio $\mathrm{SNR}=10 \log _{2}\left(1 / N_{0}\right)$ for $P_{1}=4, P_{2}=0$.

As it can be seen, for small SNRs in the second hop, it is advantageous to maximize the energy transfer to the relay, which is obtained for $p=0.5$. Instead, for sufficiently large SNR in the second hop, it is optimal to maximize the information transfer to the relay, which is obtained for $p=0.25$. This demonstrates that, in a multi-hop channel with a harvesting relay, the encoder needs to fully adjust its transmission strategy depending on the quality of the second link. This calls for a larger degree of coordination than in the conventional scenario.

\section{Conclusions}

The two baseline multi-user scenarios studied in this paper complement the initial work [4]-[7] by showing that the requirements of energy and information flow have significant consequences on the design of wireless networks with multiple terminals. Recent work has reached related conclusions for a two-way communication model [11]. An interesting avenue for future work is the design of practical coding strategies that achieve a desired trade-off between energy and information transfer.

\section{Appendix A: Sketch of Converse Proof for THEOREM 1}

The proof of the converse follows the same procedure as in [10, page 89], except for the proof of the inequality $B \leq E[b(Y)]$. To prove this bound, note that any $\left(2^{n R_{1}}, 2^{n R_{1}}, P_{1}, P_{2}, n\right)$ code needs to satisfy (4). Moreover, we have the bound

$$
\mathrm{E}\left[b^{n}\left(Y^{n}\right)\right] \geq(B-\epsilon) \operatorname{Pr}\left[b^{n}\left(Y^{n}\right) \geq B-\epsilon\right],
$$

and thus, by (4), we also have that $\mathrm{E}\left[b^{n}\left(Y^{n}\right)\right] \geq B-\epsilon$ for $n$ large enough. Given that $\mathrm{E}\left[b^{n}\left(Y^{n}\right)\right]=\frac{1}{n} \sum_{i=1}^{n} \mathrm{E}\left[b\left(Y_{i}\right)\right]$, defining $Y$ as in [10, page 81] concludes the proof.

\section{APPEndix B: Sketch of CONVERSE ProOf FOR THEOREM 1}

From the cut-set bound in [10, Theorem 16.1], we have the inequality

$$
R \leq \max _{p\left(x_{1}, x_{2}\right)} \min \left\{I\left(X_{1} ; Y_{1}\right), I\left(X_{2} ; Y_{2}\right)\right\} .
$$

We recall that this is proved by defining $Q \sim \operatorname{Unif}[1: n]$ independent of $\left(X_{1}^{n}, X_{2}^{n}\right)$ and by setting $X_{1}=X_{1 Q}, X_{2}=$ $X_{2 Q}$. With these definitions, from (9) and (10), we get $\mathrm{E}\left[c_{1}\left(X_{1}\right)\right] \leq P_{1}$ and $\mathrm{E}\left[c_{2}\left(X_{2}\right)\right] \leq \mathrm{E}\left[b\left(Y_{1}\right)\right]+P_{2}$, respectively. We then observe that the two terms inside the min function are separately functions of $p\left(x_{1}\right)$ and $p\left(x_{2}\right)$, so that we can maximize over the marginals $p\left(x_{1}\right)$ and $p\left(x_{1}\right)$. The order of the optimizations in (14) follows the same arguments.

\section{REFERENCES}

[1] V. Chawla and S. H. Dong, "An overview of passive RFID," IEEE Commun. Magazine, vol. 45, no. 9, pp. 11-17, Sept. 2007.

[2] F. Zhang, S. A. Hackworth, X. Liu, H. Chen, R. J. Sclabassi, and M. Sun, "Wireless energy transfer platform for medical sensors and implantable devices," in Proc. Annual Int. Conf. IEEE Engineering in Medicine and Biology Society (EMBC 2009), pp. 1045-1048, Sept. 2009.

[3] H. Matsumoto, "Research on solar power satellites and microwave power transmission in Japan," IEEE Microwave Magazine, vol. 3, no. 4, pp. 36- 45, Dec. 2002.

[4] L. R. Varshney, "Transporting information and energy simultaneously," in Proc. IEEE Int. Symposium on Inform. Theory (ISIT 2008), pp. 16121616, Toronto, Canada, Jul. 2008.

[5] P. Grover and A. Sahai, "Shannon meets Tesla: Wireless information and power transfer," in Proc. IEEE Int. Symposium on Inform. Theory (ISIT 2010), pp. 2363-2367, Austin, TX, Jun. 2010.

[6] R. Zhang and C. Keong, "MIMO broadcasting for simultaneous wireless information and power transfer," arXiv:1105.4999

[7] L. R. Varshney, "On energy/information cross-layer architectures," in Proc. IEEE Int. Symposium on Inform. Theory (ISIT 2012), Cambridge, MA, Jul. 2012.

[8] M. Gastpar, "On capacity under receive and spatial spectrum-sharing constraints," IEEE Trans. Inform. Theory, vol. 53, no. 2, pp. 471-487, Feb. 2007.

[9] B. Gurakan, O. Ozel, J. Yang and S. Ulukus, "Energy cooperation in energy harvesting wireless communications," in Proc. IEEE Int. Symposium on Inform. Theory (ISIT 2012), Cambridge, MA, Jul. 2012.

[10] A. El Gamal and Y.-H. Kim, Network Information Theory, Cambridge University Press, 2011.

[11] P. Popovski and O. Simeone, "Two-Way Communication with Energy Exchange," in Proc. IEEE Information Theory Workshop (ITW 2012), Lausanne, Switzerland, Sept. 3-7, 2012. 